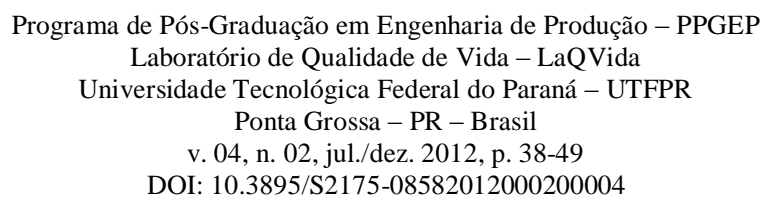

\author{
REVISTA BRASILEIRA DE \\ QUALIDADE DE VIDA
}

\title{
Qualidade de vida dos graduandos de enfermagem: análise da produção científica
}

\section{Quality of life of undergraduate nursing students: production analysis}

\author{
Camila Scarel Rosa \\ Universidade Federal de São Paulo - UNIFESP - São Paulo - Brasil \\ mila_sr@msn.com \\ Grazielle Viola Arronqui \\ Universidade Federal de São Paulo - UNIFESP - São Paulo - Brasil \\ grazi.viola@gmail.com
}

Doutoranda em Ciências pelo Programa de Pós-Graduação da Escola Paulista de Enfermagem da Universidade Federal de São Paulo

Universidade Federal de São Paulo - UNIFESP - São Paulo - Brasil

rose.lacava@unifesp.br

Solange Maria Fustinoni Magalhães

Centro Universitário Assunção da UNIFAI e Centro Universitário Sant'Anna - São Paulo - Brasil somag@terra.com.br

Rosely Erlach Goldman

Universidade Federal de São Paulo - UNIFESP - São Paulo - Brasil rosely.goldman@unifesp.br

\begin{abstract}
Resumo
O presente estudo tem por objetivo analisar a produção científica contida nas bases Medical Literature Analysis and Retrieval System On-line - MEDLINE, Scientific Eletronic Library On-line - SciELO e Literatura Latino-Americana e do Caribe em Ciências da Saúde - LILACS, no período de 1999 até 2011, sobre a qualidade de vida do graduando de enfermagem, nas dimensões metodológicas, satisfação da qualidade de vida e fatores influentes. Trata-se de um estudo descritivo de revisão sistemática da literatura. Dez artigos perfizeram o corpus documental deste estudo. Os principais resultados mostram que na maioria dos estudos optou-se pelo método descritivo com abordagem quantitativa, utilizaram o instrumento WHOQOL-bref, tendo sido publicados em periódicos avaliados com QUALIS A2 e baixo fator de impacto. Quanto à satisfação, observou-se que o graduando de enfermagem possui uma percepção positiva de sua qualidade de vida, apesar dos estudos descreverem situações de estresse, insegurança e inserção a pratica como fatores capazes de influenciar negativamente a qualidade de vida do graduando durante sua experiência acadêmica.
\end{abstract}

Palavras-chave: qualidade de vida, estudantes de enfermagem, enfermagem.

\section{Abstract}

This study aims to examine the scientific bases contained in the Medical Literature Analysis and 
Retrieval System Online - MEDLINE, Scientific Electronic Library Online - SciELO and Latin American and Caribbean Center on Health Sciences - LILACS period 1999 to 2011 about quality of life of nursing undergraduate students in methodological dimensions, satisfaction, quality of life and further influencing factors. This is a descriptive study of systematic literature review. Ten items totaled the documentary corpus of this study. The main results show that most studies were built under descriptive method with quantitative approach, using the WHOQOL-bref, being published in journals evaluated with QUALIS A2 and low impact factor. Regarding satisfaction, it was observed that the nursing undergraduate students have a positive perception of their quality of life, despite studies describing situations of stress, insecurity and the insertion practices as factors that may negatively influence the quality of life of students during their academic experience.

Keywords: quality of life, nursing students, nursing.

\section{Introdução}

O tema qualidade de vida - QV vem sendo pesquisado em diferentes áreas de conhecimento. Primeiro foi utilizado como descritor de aquisição de bens. Com o passar dos anos foi ampliado e, na atualidade, alcançou aspectos subjetivos, sendo constituído de inúmeras competências e grandezas: ambientais, físicas, psicológicas, espirituais, entre outras (KAWAKAME, MIYADAHIRA, 2005; EURICH, KLUTHCOVSKY, 2008).

É importante destacar a distinção entre padrão de vida e QV. O primeiro utiliza indicadores globais, do modo de viver de cada sociedade e indivíduo, abrangendo aspectos socioeconômicos, como poder de compra, demográficos e de cuidados de saúde oferecidos (PANZINI et al., 2007). Já o segundo, inclui, além dos aspectos objetivos, os subjetivos, como a percepção do indivíduo de sua posição na vida, no contexto da cultura e nos sistemas de valores nos quais vive e, em relação aos seus objetivos, expectativas, padrões e preocupações, ou seja, está sempre sob a percepção de quem está sendo avaliado (FLECK et al., 1999; PANZINI et al., 2007).

O tema QV tem sido reforçado como promotor de saúde e do autocuidado desde a década de 1970 (PANZINI et al., 2007), uma vez que se entendeu a saúde como um conceito amplo: "um estado de completo bem-estar físico, mental e social e não apenas a ausência de doença ou enfermidade", como estabelecido pela Organização Mundial de Saúde - OMS (FLECK et al., 1999).

Apesar disso, devido à complexidade que abrange o tema $\mathrm{QV}$, o consenso de sua definição ainda não foi obtido, o que não abrevia a sua importância no contexto da manutenção e da promoção de saúde. Igualmente, a OMS construiu um conceito que, mesmo não sendo consensual, tornou-se amplamente utilizado para QV, constituído de três aspectos fundamentais: subjetividade; multidimensionalidade; presença de dimensões positivas e negativas: a "percepção do indivíduo de sua posição na vida no contexto da cultura e sistema de valores nos quais ele vive e em relação aos seus objetivos, expectativas, padrões e preocupações" (WHOQOL GROUP, 1994; FLECK et al., 1999).

No meio acadêmico, para o estudante, a experiência universitária torna-se um momento de transição para a vida adulta, sujeito às mudanças culturais, novas expectativas, objetivos e preocupações. É quando o graduando irá se desenvolver tanto profissionalmente quanto pessoalmente. Nessa nova fase, o estudante se depara com uma nova realidade e novas exigências, que inúmeras vezes estarão fora de sua zona de conforto, o que exigirá dele uma nova postura e mudança de comportamento e, por conseguinte, pode lhe proporcionar a vivência de inúmeros conflitos (CAPLAN, 1980; FIGUEIREDO; OLIVEIRA, 1995; CORREIA, 2003).

Estudos como os de Saupe (2002), Kawakame e Miyadahira (2005), Sousa e Marques (2010) e Arronqui et al. (2011) demonstram a existência de situações desfavoráveis à QV dos graduandos de enfermagem. Pois, além de ter que se adaptar a essa fase da vida, podendo enfrentar obstáculos financeiros, familiares e de saúde, o discente terá, na sua introdução à prática, que vivenciar momentos de dor, de sofrimento e de morte. Tais situações de enfrentamento podem gerar sentimentos de medo, de ansiedade e de estresse, em razão de sua inexperiência de vida (SAUPE, 
A reflexão sobre a QV do graduando de enfermagem é um importante recurso para identificar fatores que afetam sua integridade física e mental e processos que podem favorecer ou não sua formação (KAWAKAME; MIYADAHIRA, 2005; OLIVEIRA; CIAMPONE, 2006).

O presente estudo teve como objetivo analisar a produção científica contida nas bases SciELo, MEDLINE e LILACS, no período de 1999 até 2011, sobre a QV do graduando de enfermagem, nas dimensões metodológicas, satisfação da QV e fatores influentes na QV.

\section{Método}

Trata-se de um estudo do tipo descritivo, de revisão sistemática da literatura, que adotou as seguintes etapas para a seleção da amostra: escolha das questões temática, estabelecimento de critérios de inclusão e exclusão de artigos, definição das informações a serem extraídas, análise e interpretação dos resultados e apresentação da revisão (MENDES; SILVEIRA; GALVÃO, 2008; GANONG, 1987).

Para a construção do corpus documental foram utilizadas as bases de dados da Medical Literature Analysis and Retrieval Sistem On-line - MEDLINE, da Scientific Eletronic Library Online - SciELO e da Literatura Latino-Americana e do Caribe em Ciências da Saúde - LILACS.

A questão norteadora elaborada para a seleção dos artigos foi: como é a QV do graduando de enfermagem? Descritores utilizados: língua portuguesa: "qualidade de vida", "estudantes de enfermagem" e "enfermagem"; língua espanhola: "calidad de vida", "estudiantes de enfermería" e "enfermería"; língua inglesa: "quality of life", "students, nursing" e "nursing". Nas bases de dados recorreu-se aos operadores lógicos "and", "or" e "and not", para combinação dos descritores e rastreamento das produções científicas.

Foram definidos os seguintes critérios de inclusão:

a) artigos publicados em português, em inglês e em espanhol, no período de janeiro de 1999 a dezembro de 2011;

b) artigos publicados na integra;

c) com graduandos de enfermagem, como sujeitos dos estudos;

d) temática de forma clara no título e no resumo.

Excluíram-se os artigos repetidos.

Encontrou-se 479 artigos, a saber: 53 na língua portuguesa, 238 na língua inglesa e 52 na língua espanhola, na base MEDLINE; 8 artigos em português, 23 em inglês e 63 em espanhol na SciELO; e 11 artigos em português, 6 em inglês e 25 em espanhol na LILACS.

Em seguida foram avaliados os títulos e os resumos dos 479 artigos e, desses, 467 foram excluídos por não responderem a questão norteadora. Posteriormente, dois trabalhos foram excluídos, por terem disponibilizado apenas o resumo. Ao final, a amostra foi composta por nove artigos na língua portuguesa e um na língua espanhola, totalizando dez artigos selecionados, os quais foram lidos na íntegra.

Os estudos foram analisados, sendo observados os fatores de adequação da metodologia empregada e delineamento da pesquisa segundo: clareza na definição do objetivo, seleção randomizada da amostra, utilização de instrumentos validados ou não e apresentação de fatores influentes na QV do graduando de enfermagem.

\section{Resultados e discussões}

\subsection{Apresentação do corpus documental}

O Quadro 1 apresenta aspectos metodológicos dos artigos que compõem o corpus documental do estudo: 
Quadro 1 - Corpus documental

\begin{tabular}{|c|c|c|c|c|c|}
\hline \multirow{2}{*}{ TÍTULO / DATA / PERIÓDICO } & \multirow{2}{*}{$\begin{array}{c}\text { DELINEAMENTO DO } \\
\text { ESTUDO / INSTRUMENTO }\end{array}$} & \multirow{2}{*}{$\begin{array}{l}\text { POPULAÇÃO/ } \\
\text { AMOSTRA }\end{array}$} & \multicolumn{3}{|c|}{$\begin{array}{c}\text { CARACTERIZAÇÃO DA } \\
\text { AMOSTRA }\end{array}$} \\
\hline & & & SEXO & IDADE & \begin{tabular}{|c|} 
ESTADO \\
CIVIL \\
\end{tabular} \\
\hline $\begin{array}{l}\text { 1. Qualidade de vida de estudantes de } \\
\text { enfermagem conforme escala de } \\
\text { Flanagan / 2002/ Revista Ciência, } \\
\text { Cuidado e Saúde. }\end{array}$ & $\begin{array}{l}\text { Estudo descritivo de abordagem } \\
\text { quantitativa. } \\
\text { - } \quad \text { Escala de Flanagan. }\end{array}$ & $100 / 86$ & $\begin{array}{c}92 \% \\
\text { feminino. }\end{array}$ & $20-30$ & $\begin{array}{c}91 \% \\
\text { solteiros. }\end{array}$ \\
\hline $\begin{array}{l}\text { 2. Qualidade e estilo de vida de } \\
\text { estudantes de medicina e de } \\
\text { enfermagem / 2004/ Revista do } \\
\text { Hospital de Psiquiatria de La Habana. }\end{array}$ & $\begin{array}{l}\text { Estudo descritivo de abordagem } \\
\text { quantitativa. } \\
\text { - Índice de qualidade de } \\
\text { vida - IQV. } \\
\text { - } \quad \text { Questionário geral de } \\
\text { saúde (autoria anônima). }\end{array}$ & $69 / 68$ & $\begin{array}{c}63 \% \\
\text { feminino. }\end{array}$ & $22-24$ & $\begin{array}{c}76,5 \% \\
\text { solteiros. }\end{array}$ \\
\hline $\begin{array}{l}\text { 3. Qualidade de vida dos acadêmicos } \\
\text { de enfermagem / 2004/ Revista } \\
\text { Latino-Americana de Enfermagem. }\end{array}$ & $\begin{array}{l}\text { Estudo descritivo de abordagem } \\
\text { quantitativa. } \\
\text { - } \quad \text { WHOQOL bref. }\end{array}$ & $1012 / 825$ & $\begin{array}{c}90 \% \\
\text { feminino. }\end{array}$ & $17-38$ & $\begin{array}{c}83 \% \\
\text { solteiros. }\end{array}$ \\
\hline $\begin{array}{l}\text { 4. Qualidade de vida de estudantes de } \\
\text { graduação em enfermagem / 2005/ } \\
\text { Revista da escola de enfermagem da } \\
\text { USP. }\end{array}$ & $\begin{array}{l}\text { Estudo descritivo de abordagem } \\
\text { quantitativa. } \\
\text { IQV. }\end{array}$ & $264 / 173$ & $\begin{array}{l}89,4 \% \\
\text { feminino. }\end{array}$ & $|17-40|$ & $\begin{array}{c}88,6 \% \\
\text { solteiros. }\end{array}$ \\
\hline $\begin{array}{l}\text { 5. Qualidade de vida de estudantes de } \\
\text { enfermagem: a construção de um } \\
\text { processo e intervenções / 2008/ } \\
\text { Revista da escola de enfermagem da } \\
\text { USP }\end{array}$ & $\begin{array}{l}\text { Pesquisa qualitativa na } \\
\text { modalidade pesquisa-ação- } \\
\text { grupo focal associada a alguns } \\
\text { elementos da técnica de grupo } \\
\text { operativo. } \\
\text { - Grupo focal com } \\
\text { discussões sobre o tema. }\end{array}$ & $-/ 11$ & - & - & - \\
\hline $\begin{array}{l}\text { 6. Avaliação da qualidade de vida de } \\
\text { acadêmicos de graduação em } \\
\text { enfermagem do primeiro e do quarto } \\
\text { anos: influência das variáveis } \\
\text { sociodemográficas / 2008/ Revista de } \\
\text { psiquiatria do Rio Grande do Sul. }\end{array}$ & $\begin{array}{l}\text { Estudo descritivo, transversal e } \\
\text { de abordagem quantitativa. } \\
\text { WHOQOL-bref. } \\
\text { - } \quad \text { questionário } \\
\text { sociodemográfico (autoria } \\
\text { própria). }\end{array}$ & $-/ 67$ & $\begin{array}{l}80,6 \% \\
\text { feminino. }\end{array}$ & $17-44$ & $\begin{array}{c}90 \% \\
\text { solteiros. }\end{array}$ \\
\hline $\begin{array}{l}\text { 7. Depressão entre estudantes de dois } \\
\text { cursos de enfermagem: } \\
\text { autoavaliação da saúde e fatores } \\
\text { associados / 2010/ Revista brasileira } \\
\text { de enfermagem. }\end{array}$ & $\begin{array}{l}\text { Pesquisa com técnicas } \\
\text { qualitativas e quantitativas, bem } \\
\text { como a interface entre elas. } \\
\text { - } \quad \text { WHOQOL - bref. } \\
\text { Critérios de } \\
\text { Classificação Econômica Brasil. } \\
\text { Inventário de Depressão } \\
\text { de Beck. }\end{array}$ & $134 / 114$ & $\begin{array}{c}82,5 \% \\
\text { feminino. }\end{array}$ & $20-24$ & $\begin{array}{c}91,2 \% \\
\text { solteiros. }\end{array}$ \\
\hline $\begin{array}{l}\text { 8. Período de estudos e qualidade de } \\
\text { vida do estudante de enfermagem } \\
\text { 2010/ Revista Brasileira de Qualidade } \\
\text { de Vida }\end{array}$ & $\begin{array}{l}\text { Estudo correlacional de } \\
\text { abordagem quantitativa. } \\
\text { WHOQOL-bref. }\end{array}$ & $-/ 230$ & $\begin{array}{c}81,7 \% \\
\text { feminino. }\end{array}$ & $\begin{array}{l}<20- \\
>40\end{array}$ & - \\
\hline $\begin{array}{l}\text { 9. Qualidade de vida de estudantes de } \\
\text { enfermagem de uma faculdade privada } \\
\text { 2010/ Revista Brasileira de } \\
\text { Qualidade de Vida }\end{array}$ & $\begin{array}{l}\text { Estudo descritivo de abordagem } \\
\text { quantitativa. } \\
\text { Escala de Flanagan }\end{array}$ & $243 / 194$ & $\begin{array}{c}80 \% \\
\text { feminino }\end{array}$ & $17-53$ & $\begin{array}{c}70 \% \\
\text { solteiros }\end{array}$ \\
\hline $\begin{array}{l}\text { 10. Percepção de graduandos de } \\
\text { enfermagem sobre sua qualidade de } \\
\text { vida / 2011/ Acta Paulista de } \\
\text { Enfermagem }\end{array}$ & $\begin{array}{l}\text { Estudo descritivo, com desenho } \\
\text { transversal e abordagem } \\
\text { quantitativa. } \\
\text { - } \quad \text { WHOQOL-bref }\end{array}$ & $272 / 178$ & - & - & - \\
\hline
\end{tabular}

Fonte: Autoria própria (2012).

Os estudos foram publicados no período de 2002 a 2011, sendo a maioria $(n=6)$ realizada no Estado de São Paulo ( $n=5)$, com delineamento quantitativo descritivo ( $n=7)$.

Evidenciou-se que na totalidade dos estudos a maioria dos entrevistados é do sexo feminino 
e de estado civil solteira, sendo que a idade variou entre 17 e 53 anos. Havendo uma referência histórica à predominância do sexo feminino na profissão de enfermagem (DAUBERMANN; TONETE, 2012; FERNANDES et al., 2012).

É evidente a pouca quantidade de estudos publicados sobre a QV do graduando de enfermagem e também a concentração dos estudos realizados nas regiões mais desenvolvidas do país deve-se à maior concentração de cursos de graduação e de pós-graduação nessa região.

A escolha predominante do desenho descritivo na modalidade quantitativa entre a maioria dos trabalhos é justificada pelo anseio de uma pesquisa capaz de quantificar e descrever características e aspectos semiológicos, etiológicos, fisiopatológicos e epidemiológicos de uma situação. Este procedimento pode, também, ser utilizado para conhecer uma situação, estudando a sua distribuição no tempo (período), no espaço (associação com a localidade) e conforme peculiaridades individuais (sexo, idade, condições socioeconômicas) (HOCHMAN, 2012).

Quanto ao instrumento empregado para avaliar a QV dos graduandos, constatou-se que o mais utilizado $(n=5)$ foi o WHOQOL-bref. Todavia, estudo de Saupe et al. (2004) recomenda a utilização complementar de uma entrevista semiestruturada para possibilitar uma intervenção individualizada, como realizado nas pesquisas de Eurich e Kluthcovsky (2008) e Furegato, Ferreira e Silva (2010).

A maior utilização do WHOQOL-bref foi atribuída, além de sua fácil utilização, à sua validação no Brasil (SOUZA, MARQUES, 2010) ou ao seu modo sistemático e pelo caráter internacional e transcultural do desenvolvimento do instrumento, permitindo, portanto, o desenvolvimento de pesquisas em diversos centros temáticos e a comparação dos resultados entre diferentes populações (EURICH, KLUTHCOVSKY, 2008; FUREGATO, FERREIRA e SILVA, 2010).

Contudo, a avaliação da QV por meio de um mesmo instrumento produz um panorama situacional limitado, sendo necessária a realização de estudos aprofundados que utilizem diferentes instrumentos, capazes de investigar a temática e vieses distintos, uma vez que as situações estressantes e os fatores que podem influenciar a QV dos graduandos de enfermagem em sua vivência acadêmica deixam de ser percebidos com a uniformização instrumental, como afirmam Oliveira e Ciampone (2008).

Os dois estudos que utilizaram a Escala de Qualidade de Vida - EQV de Flanagan versavam sobre itens conceituais da QV, sendo representados em cinco dimensões: bem-estar físico e material, relação com outras pessoas, atividades sociais, comunitárias e cívicas, desenvolvimento pessoal e realização e recreação. A EQV foi desenvolvida nos Estados Unidos em 1982, e não possui uma versão validada para a cultura brasileira, tendo sido traduzida para o português por Hashimoto e colaboradores (1996), os quais não fizeram nenhuma referência quanto a sua fidedignidade (SANTOS, 2002).

Já o Índice de Qualidade de Vida - IQV, um instrumento genérico, utilizado em duas pesquisas, que pode ser aplicado tanto em pessoas sadias como em doentes, foi traduzido e validado para o português do Brasil em 1999, sendo excluídos 7 dos 68 itens do IQV original, divididos pelos domínios: saúde, funcionamento, socioeconômico, psicológico, espiritual e família (KAWAKAME; MIYADAHIRA, 2005).

O Quadro 2 apresenta a avaliação CAPES dos periódicos nos quais foram publicadas as pesquisas deste estudo: 
Quadro 2 - Periódicos de publicação, avaliação CAPES em 2012, na área de enfermagem (em atualização) e fator de impacto

\begin{tabular}{|c|c|c|c|}
\hline PERIÓDICO & $\begin{array}{c}\text { QUANTIDADE DE } \\
\text { ARTIGOS PUBLICADOS }\end{array}$ & QUALIS & $\begin{array}{c}\text { FATOR DE IMPACTO } \\
\text { (Journal Citation Reports - } \\
\text { JCR 2011) }\end{array}$ \\
\hline $\begin{array}{c}\text { Revista Ciência, Cuidado e } \\
\text { Saúde }\end{array}$ & 1 & B1 & Não consta \\
\hline $\begin{array}{c}\text { Revista do Hospital } \\
\text { Psiquiátrico de La Habana }\end{array}$ & 1 & $\begin{array}{c}\text { Não } \\
\text { consta }\end{array}$ & Não consta \\
\hline $\begin{array}{c}\text { Revista Latino-Americana } \\
\text { de Enfermagem }\end{array}$ & 1 & A2 & 0,625 \\
\hline $\begin{array}{c}\text { Revista da Escola de } \\
\text { Enfermagem da USP }\end{array}$ & 2 & A2 & Não consta \\
\hline $\begin{array}{c}\text { Revista Brasileira de } \\
\text { Enfermagem }\end{array}$ & 1 & - & Não consta \\
\hline $\begin{array}{c}\text { Revista de Psiquiatria do } \\
\text { Rio Grande do Sul }\end{array}$ & 2 & - & Não consta \\
\hline $\begin{array}{c}\text { Revista Brasileira de } \\
\text { Qualidade de Vida }\end{array}$ & 1 & A2 & 0,204 \\
\hline $\begin{array}{c}\text { Acta Paulista de } \\
\text { Enfermagem }\end{array}$ & 1 & Ang \\
\hline
\end{tabular}

A classificação apresentada no quadro não corresponde ao momento da publicação.

Fonte: Autoria própria (2012).

A maioria dos artigos $(n=4)$ foi publicada em periódicos classificados nos estratos superiores do QUALIS (A2), numa escala decrescente de A1, A2, B1, B2, B3, B4, B5 a C, na qual B3, B4 e B5 não possuem valor de impacto por se tratar de bases de dados (ANDRIOLO, 2010). Não obstante, existem questionamentos importantes, assinados por editores de diferentes periódicos, quanto aos critérios empregados pela CAPES para tal avaliação, sendo o fator de impacto (JCR) o mais relevante. Entre as questões levantadas estão a não observância dos diferenciais existentes no parque editorial brasileiro, que é mantido basicamente pela iniciativa privada e/ou por universidades públicas e privadas, bem como, a falta de incentivo financeiro e a necessidade de maior visibilidade para os periódicos nacionais no exterior (ANDRIOLO, 2010). Entretanto, não havendo outros meios de avaliação, o índice de JCR deve ser considerado um bom recurso técnico de avaliação científica, tendo grande influência na vida acadêmica e científica nacional (RUIZ; GRECO; BRAILE, 2009).

Por ser uma medida estatística, que considera o número de vezes que um artigo foi citado em um determinado período, o JCR pode ser afetado e ter o seu valor interpretado erroneamente (MARZIALE, MENDES, 2002). Apenas três periódicos que compõem o corpus documental do estudo possuem avaliação do JCR, mesmo assim, obtiveram baixo valor de fator de impacto (inferior a 0,7). Entretanto um baixo JCR não é necessariamente indicador da qualidade científica, uma vez que, diferentes áreas têm diferentes práticas de investigação.

Ao mesmo tempo em que se observa a avaliação QUALIS dos periódicos em estratos superiores, é visível o baixo índice do JCR, evidenciando que a produção qualificada na área de enfermagem não alcança difusão internacional, principalmente em função da língua portuguesa.

\subsection{Fatores influentes na QV dos graduandos de enfermagem}

O Quadro 3 apresenta a avaliação e fatores influentes na QV das produções científicas: 
Quadro 3 - Avaliação da qualidade de vida

\begin{tabular}{|c|c|c|c|}
\hline \multirow{2}{*}{ N. ${ }^{\circ}$} & \multirow{2}{*}{ AVALIAÇÃO DA QV } & \multicolumn{2}{|c|}{ FATORES INFLUENTES NA QV } \\
\hline & & COTIDIANO & MEIO ACADÊMICO \\
\hline 1 & $\begin{array}{l}\text { Escala de Flanaganm } \\
\text { 42,5\% satisfeitos. }\end{array}$ & - & \begin{tabular}{|l} 
Positivos: \\
- $\quad$ Possibilidade de ajudar os outros; \\
- $\quad$ Aprendizagem da humanização; \\
- Amadurecimento; \\
- $\quad$ Melhora de hábitos; \\
- Sensação de produtividade e \\
- $\quad$ Realização pessoal; \\
- Satisfação. \\
Negativos: \\
- Volume da carga horária; \\
- Necessidade de ter de trabalhar; \\
- Estresse.
\end{tabular} \\
\hline 2 & $\begin{array}{ll}\text { - } & \text { IQV. } \\
\text { - } & \text { Questionário geral de } \\
& \text { saúde. } \\
& \\
\text { Média } 7 \text { em escala de } 1 \text { a } 10 \mathrm{~m}\end{array}$ & 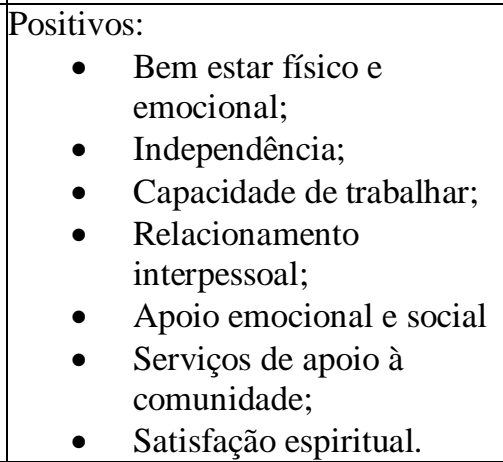 & - \\
\hline 3 & $\begin{array}{l}\text { - WHOQOL-bref } \\
64 \% \text { satisfeitos. }\end{array}$ & \begin{tabular}{|l}
\multicolumn{2}{|l}{ Negativos: } \\
- \\
- \\
- Ansieenças crônicas; \\
- Insônia; \\
- \\
Cansaço crônico; \\
Estresse.
\end{tabular} & \begin{tabular}{|ll} 
Negativos: \\
- & Irritação; \\
- & Desânimo e/ou cansaço; \\
- & Discussão com amigos e com \\
& familiares; \\
- & Pensamentos que provocam ansiedade \\
& e esgotamento emocional.
\end{tabular} \\
\hline 4 & \begin{tabular}{|c|} 
IQV \\
Média 25,4 em escala de 1 a 30.
\end{tabular} & 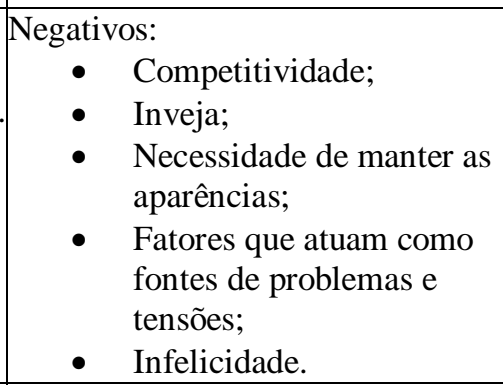 & $\begin{array}{l}\text { Negativos: } \\
\text { • Inserção do aluno em campo prático. }\end{array}$ \\
\hline 5 & \begin{tabular}{|l} 
Grupo focal com \\
discussões sobre o \\
tema.
\end{tabular} & - & \begin{tabular}{|ll} 
Negativos: \\
- & Falta de acolhimento quanto às \\
& questões internas; \\
- & Relação professor-aluno; \\
- & Situações geradoras de medo; \\
- & Insegurança; \\
- & Pressão.
\end{tabular} \\
\hline 6 & 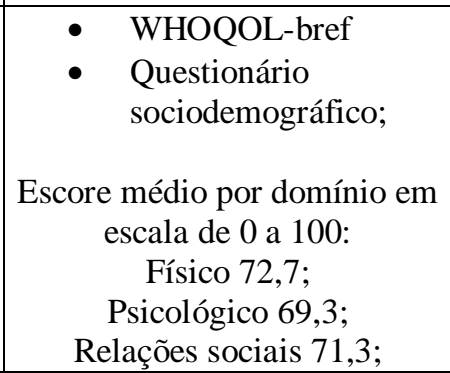 & - & \begin{tabular}{|l} 
Negativos: \\
- $\quad$ Falta de acolhimento por parte dos \\
professores e enfermeiros de campo; \\
- $\quad$ Falta de integração com equipe e \\
alunos de outros cursos; \\
- Carga horária excessiva para o aluno \\
trabalhador.
\end{tabular} \\
\hline
\end{tabular}




\begin{tabular}{|c|c|c|c|}
\hline & Meio ambiente $60,7$. & & \\
\hline 7 & 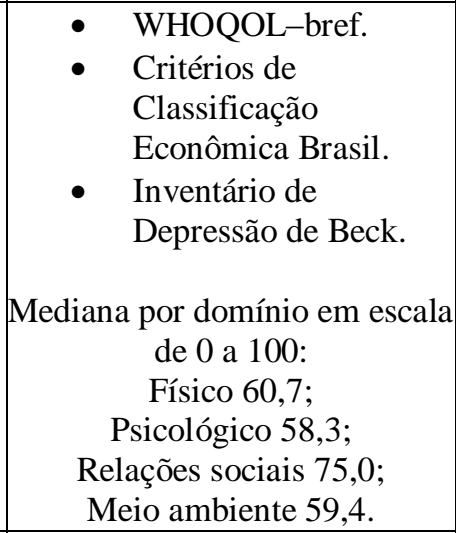 & \begin{tabular}{|l} 
Negativos: \\
- $\quad$ Depressão; \\
- $\quad$ Classe social; \\
- $\quad$ Trabalho remunerado.
\end{tabular} & $\begin{array}{l}\text { Negativos: } \\
\text { • Tipo de curso (licenciatura). }\end{array}$ \\
\hline 8 & $\begin{array}{c}\text { WHOQOL-bref. } \\
\text { Média da QV segundo período } \\
\text { de estudo: } \\
\text { Manhã } 52,5 ; \\
\text { Noite } 49,6 .\end{array}$ & \begin{tabular}{|l} 
Negativos: \\
- $\quad$ Trabalho remunerado; \\
- $\quad$ Sedentarismo.
\end{tabular} & \begin{tabular}{|l} 
Negativos: \\
- \\
Preocupação com o mercado de \\
trabalho; \\
- Aumento da tensão ou do estresse \\
psíquico; \\
- Sobrecarga da carga horária em sala de \\
- \\
aula; \\
Necessidade de despender seu tempo \\
relacionadas à universidade ou aos \\
estudos; \\
Sentimentos negativos ao final do \\
curso.
\end{tabular} \\
\hline 9 & $\begin{array}{l}\text { Escala de Flanagan. } \\
\text { 34,3\% satisfeitos. }\end{array}$ & \begin{tabular}{|l} 
Negativos: \\
- $\quad$ Mudança no padrão de \\
- \\
- Ritmo; \\
- Dificuldades vida acelerado; \\
um emprego; \\
- Desgaste físico e emocional; \\
- Pouco tempo para lazer e \\
\\
família.
\end{tabular} & \begin{tabular}{|l} 
Positivos: \\
- $\quad$ Realização de um sonho; \\
- $\quad$ Pjudar a pessoas; \\
- $\quad$ Sentimectiva de um futuro melhor; \\
- Interação do curso com o cotidiano; \\
- $\quad$ Conhecimento.
\end{tabular} \\
\hline 10 & $\begin{array}{l}\text { WHOQOL-bref } \\
\text { Escore médio por domínio em } \\
\text { escala de } 0 \text { a } 100: \\
\text { Físico } 48,3 ; \\
\text { Psicológico } 61,9 . \\
\text { Relações sociais } 71,1 \\
\text { Meio ambiente } 57,0 .\end{array}$ & 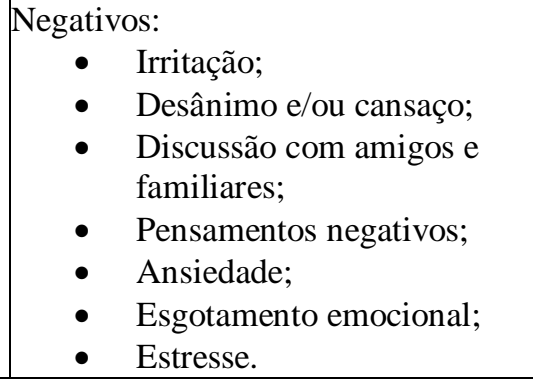 & \begin{tabular}{|l} 
Negativos: \\
- Falta de espaços de acolhimento e de \\
- \\
lazer; \\
Falta de suporte para enfrentamento \\
- \\
Falta dituações vividas \\
- desenvolvidas; \\
- $\quad$ Sobrecarga horária das atividades do curso; \\
Relaço professor-estudante.
\end{tabular} \\
\hline
\end{tabular}

Fonte: Autoria própria (2012).

Observa-se que, apesar da maioria dos estudos apontarem resultados positivos quanto à avaliação da QV na graduação, são descritos inúmeros fatores preocupantes, capazes de influenciar negativamente a QV, tanto no cotidiano quanto no meio acadêmico: estresse, insegurança e não reconhecimento das atividades executadas durante a formação, conflitos de enfrentamento de situações profissionais e a preocupação com o mercado de trabalho, falta de tempo para prática de lazer, necessidade de ter de trabalhar durante o curso, entre outros (KAWAKAME, MIYADAHIRA, 2005; IGLESIAS, 2002; SAUPE, 2002).

De forma adicional, o estudo de Iglesias (2002) aponta como aspecto interferente da QV o fato de que quase a totalidade dos alunos-trabalhadores estava empregada em hospitais, exercendo a função de técnico ou de auxiliar de enfermagem, e dedicavam aos estudos menos de duas horas semanais, queixando-se de não conseguir manter a atenção nas aulas devido a cansaço, estresse e 
sonolência.

Contudo, o estudo de Furegato, Ferreira e Silva (2010) apresenta que a depressão é menos frequente entre alunos que trabalham. Esses autores não encontraram diferenças estatisticamente significativas na QV dos graduandos pelo fato de trabalharem ou não.

Já na pesquisa de Kawakame e Miyadahira (2005) os resultados da avaliação da QV, evidenciou ser menos favorável no $2^{\circ}$ ano da graduação, e foram atribuídos à inserção do aluno em campo prático, uma vez que proporcionou a geração de novos conflitos, de mudanças no cotidiano, novas experiências e sentimentos. Outros autores como Oliveira e Ciampone (2008), Alves (2010) e Arronqui et al. (2011) encontraram resultados semelhantes e são unânimes ao concordar com a situação.

Para Oliveira e Ciampone (2006), a universidade possui dois polos, favorável e desfavorável, à promoção da $\mathrm{QV}$, logo, defende a "[...] necessidade de preparo dos futuros profissionais de saúde e dos docentes para o desenvolvimento do cuidado de si, como precedente da condição para o cuidar de outros”. Sousa e Marques (2010) e Arronqui et al. (2011) concordaram com a afirmação anterior, e também com Saupe (2002) em suas considerações e propostas de intervenções, afirmando ser indispensável à disponibilização de recursos de apoio individual ao estudante em diferentes perspectivas.

Tais aspectos, associados ao volume de carga horária, muitas vezes em período integral, à pressão e à exigência no ambiente acadêmico e à inexistência ou à insuficiência das redes de apoio ao aluno nas universidades, podem despertar no graduando um sofrimento emocional e psíquico interferindo na QV e levando-o a uma insatisfação pessoal (SAUPE, 2002; ARRONQUI et al., 2011).

Apenas os estudos de Saupe (2002) e de Alves (2010) citam aspectos positivos da graduação de enfermagem, ambos concordaram que a possibilidade de ajudar os outros e os conhecimentos adquiridos durante a graduação são fatores que podem influenciar positivamente a QV dos estudantes.

Quanto aos aspectos negativos, aparece entre os mais citados o relacionamento interpessoal com familiares, amigos e principalmente professor-aluno (SAUPE et al. 2004; OLIVEIRA, CIAMPONE, 2008; EURICH, KLUTHCOVSKY, 2008; ARRONQUI et al. 2011). É importante destacar que são aspectos exteriores ao meio acadêmico.

Considerando os estudos analisados, são inúmeros os fatores capazes de influenciar a QV dos graduandos de enfermagem. Por outro lado, visando uma mudança situacional, são expostas as necessidades de uma reestruturação acadêmica tanto física, que contemple melhores condições ambientais de estudo, quanto nos serviços de apoio oferecidos aos alunos, de maneira que seja possível minimizar os conflitos, os sofrimentos e as angústias que surgem no decorrer da graduação (SAUPE, 2002; OLIVEIRA, CIAMPONE, 2006; EURICH, KLUTHCOVSKY, 2008 ARRONQUI et al., 2011).

\subsection{Considerações finais}

Constatou-se que a qualidade metodológica dos estudos sobre a QV dos graduandos de enfermagem é satisfatória, apesar do número extremamente restrito de estudos. A maioria destes optou pelo método descritivo com abordagem quantitativa $(\mathrm{n}=7)$, suprindo as necessidades objetivas de cada pesquisa. A maioria dos estudos $(n=5)$ optou por utilizar um instrumento validado, o WHOQOL-bref, o que reproduziu um panorama situacional limitado, dificultando a avaliação individualizada de cada discente e impedindo a análise aprofundada do tema.

Considerando a avaliação CAPES 2012, a maioria dos periódicos foi classificada nos altos estratos QUALIS A2 ( $\mathrm{n}=4)$. Destes, apenas 3 apresentam JCR. A situação é explicada pelos padrões de exigência contidos nas bases analisadas e a abrangência limitada dos periódicos em língua portuguesa.

Por fim, pode-se afirmar que o graduando de enfermagem está satisfeito com a sua QV, 
considerando-a boa, apesar de quase a totalidade dos estudos descreverem aspectos negativos da graduação sobre a QV. Situações de estresse, insegurança, volume de carga horária e inserção a pratica, e principalmente o relacionamento interpessoal, relação professor-aluno são citados como os principais fatores capazes de influenciar negativamente a $\mathrm{QV}$ do graduando durante sua experiência acadêmica.

\section{Referências}

ALVES, E. F. Qualidade de vida de estudantes de enfermagem de uma faculdade privada. Revista Brasileira de Qualidade de Vida. Paraná, Brasil. v. 2, n. 1, p. 23-30, jan./jun. 2010.

ANDRIOLO, A. et al. Classificação dos Periódicos no Sistema QUALIS da CAPES - a Mudança dos Critérios é URGENTE!. Arquivos Brasileiro de Cardiologia, São Paulo, v. 94, n. 3, mar. 2010.

ARRONQUI, G. V. et al. Percepção de graduandos de enfermagem sobre sua qualidade de vida. Acta Paulista de Enfermagem, São Paulo, v. 24, n. 6, 2011.

CAPLAN, G. Princípios de Psiquiatria Preventiva. Rio de Janeiro: Jorge Zahar, 1980.

DAUBERMANN, D. C.; TONETE, V. L. P. Qualidade de vida no trabalho do enfermeiro da atenção básica à saúde. Acta Paulista de Enfermagem, v. 25, n. 2, p. 277-283, 2012.

EURICH, R. B.; KLUTHCOVSKY, A. C. G. C. Avaliação da qualidade de vida de acadêmicos de graduação em Enfermagem do primeiro e quarto anos: influência das variáveis sociodemográficas. Revista de Psiquiatria do Rio Grande do Sul, Porto Alegre, v. 30, n. 3, dez. 2008.

FARQUHAR M. Definitions of quality of life: a taxonomy. Journal of Advanced Nursing, v. 22, n. 3, p. 502-508, 1995.

FERNANDES, J. S. et al. A relação dos aspectos profissionais na qualidade de vida de enfermeiros das equipes saúde da família. Revista da Escola de Enfermagem da USP, v. 46, n. 2, p. 404-412, 2012.

FIGUEIREDO, R. M.; OLIVEIRA, M. A. P. Necessidades de estudantes universitários para implantação de um serviço de orientação em saúde mental. Revista Latino-Americana de Enfermagem, Ribeirão Preto, v. 3, n. 1, p. 5-18, jan./jun. 1995.

FLECK, M. P. A. et al. Desenvolvimento da versão em português do instrumento de avaliação de qualidade de vida da OMS (WHOQOL-100). Revista Brasileira de Psiquiatria, São Paulo, v. 21, n. 1, mar. 1999.

FLECK, M. P. A. et al. Aplicação da versão em português do instrumento abreviado de avaliação da qualidade de vida - "WHOQOL-bref". Revista de Saúde Pública, São Paulo, v. 34, n. 2, abr. 2000.

FUREGATO, A. R. F.; SANTOS, J. L. F.; SILVA, E. C. Depressão entre estudantes de dois cursos de enfermagem: autoavaliação da saúde e fatores associados. Revista Brasileira de Enfermagem, Brasília, v. 63, n. 4, ago. 2010.

GANONG, L. H. Integrative reviews of nursing research. Research in Nursing \& Health, v. 10, n. 1, p. 1-11, 1987. 
GONZÁLEZ M. R. et al. Calidad y estilo de vida en estudiantes de medicina y enfermeria, Revista del Hospital Psiquiátrico de La Habana, v.1, n. 1, 2004.

HOCHMAN, Bernardo et al. Desenhos de pesquisa. Acta Cir. Bras., São Paulo, 2012.

IGLESIAS, R. B. Qualidade de vida de alunos-trabalhadores que cursam a graduaçäo em enfermagem, BR41.1 - Biblioteca Wanda de Aguiar Horta BR41.1; T2140, São Paulo, 2002.

KAWAKAME, P. M. G.; MIYADAHIRA, A. M. K. Qualidade de vida de estudantes de graduação em enfermagem. Revista da Escola de Enfermagem da USP, São Paulo, v. 39, n. 2, jun. 2005.

KIMURA, M.; SILVA, J. V. Índice de qualidade de vida de Ferrans e Powers. Revista da Escola de Enfermagem da USP, São Paulo, v. 43, n. 2, p.1098-1104, 2009.

LENTZ, R. A. et al. O profissional de enfermagem e a qualidade de vida: uma abordagem fundamentada nas dimensões propostas por Flanagan. Revista Latino-Americana de Enfermagem, Ribeirão Preto, v. 8, n. 4, ago. 2000 .

MARZIALE, M. H. P.; MENDES, I. A. C.. O fator de impacto das publicações científicas. Revista Latino-Americana de Enfermagem, Ribeirão Preto, v. 10, n. 4, jul. 2002 .

MENDES, K. D. S.; SILVEIRA, PEREIRA, R. C. C. P.; GALVAO, C. M.. Revisão integrativa: método de pesquisa para a incorporação de evidências na saúde e na enfermagem. Texto Contexto Enfermagem, Florianópolis, v. 17, n. 4, dez. 2008.

OLIVEIRA, R. A.; CIAMPONE, M. H. T. A universidade como espaço promotor de qualidade de vida: vivências e expressões dos alunos de enfermagem. Texto Contexto Enfermagem, Florianópolis, v. 15, n. 2, jun. 2006 .

OLIVEIRA, R. A.; CIAMPONE, M. H. T. Qualidade de vida de estudantes de enfermagem: a construção de um processo e intervenções. Revista da Escola de Enfermagem - USP, São Paulo, v. 42, n. 1, mar. 2008 .

PANZINI, R. G. et al. Qualidade de vida e espiritualidade. Revista de Psiquiatria Clínica, São Paulo, v. 34 supl 1, p. 105-115, 2007.

RUIZ, M. A.; GRECO, O. T.; BRAILE, D. M. Fator de impacto: importância e influência no meio editorial, acadêmico e científico. Revista Brasileira de Cirurgia Cardiovascular, São José do Rio Preto, v. 24, n. 3, set. 2009 .

SANTOS, S. R. et al. Qualidade de vida do idoso na comunidade: aplicação da Escala de Flanagan. Revista Latino-Americana de Enfermagem, Ribeirão Preto, v. 10, n. 6, dez. 2002 .

SAUPE, R. Qualidade de vida de estudantes de enfermagem conforme Escala de Flanagan. Ciência, Cuidado e Saúde, Maringá,v.1,n. 2, p.287-291, jul/dez. 2002.

SAUPE, R. et al. Qualidade de vida dos acadêmicos de enfermagem. Revista Latino-Americana de Enfermagem, Ribeirão Preto, v. 12, n. 4, ago. 2004 .

SEIDL, E. M. F.; ZANNON, C. M. L. C. Qualidade de vida e saúde: aspectos conceituais e metodológicos. Caderno de Saúde Pública, Rio de Janeiro, v. 20, n. 2, abr. 2004. 
SOUSA, N. A., MARQUES, I. R. Período de estudos e qualidade de vida do estudante de enfermagem. Revista Brasileira de Qualidade de Vida. Paraná, Brasil. v. 20, n. 2, p. 01-08, jul./dez. 2010.

THE WHOQOL GROUP. The World Health Organization Quality of Life Assesment (WHOQOL): position paper the world health organization. Social Science and Medicine, v. 41, n. 10, p. 14031409, 1995.

KLUTHCOVSKY, A. C. G.C.; KLUTHCOVSKY, F. A. O WHOQOL-bref, um instrumento para avaliar qualidade de vida: uma revisão sistemática. Revista de psiquiatria do Rio Grande do Sul, Porto Alegre, v. 31, n. 3, 2009. 